\title{
The Influence of Chemical Composition of Aliphatic-Aromatic Copolyesters on Their Properties
}

\author{
Malgorzata Wojtczak ${ }^{\mathrm{a}}$, Andrzej Galeski ${ }^{\mathrm{a}}$, Slawomir Dutkiewicz ${ }^{\mathrm{b}}$, Ewa Piorkowska ${ }^{\mathrm{a}}$ \\ ${ }^{a}$ Centre of Molecular and Macromolecular Studies, Polish Academy of Sciences, Sienkiewicza 112, 90-363 \\ Lodz, Poland \\ ${ }^{b}$ Institute of Biopolymers and Chemical Fibres, Marii Sklodowskiej-Curie 19/27, 90-570 Lodz, Poland
}

\begin{abstract}
The chain microstructure and properties of a series of aliphatic-aromatic copolyesters in a range of compositions from 10 to $100 \%$ of aromatic components were studied by examining melting and crystallization behaviors, dynamic mechanical response, morphology, wide- (WAXS) and small-angle X-ray scattering (SAXS), and tensile deformation. Chain microstructure was analyzed by ${ }^{1} \mathrm{H}$ NMR. The results indicate that most of copolyesters used in this study have essentially random distribution of comonomers. Copolyesters with more than $30 \mathrm{~mol} \%$ of aromatic part crystallize with a crystal structure characteristic for homopolymer poly(butylene terephthalate) (PBT). However, some of the reflections from crystal planes are shifted towards lower diffraction angles as compared to butylene terephthalate homoplymer. The phase transition temperatures decrease with increasing aliphatic content. By means of polarized light microscopy (PLM), small-angle light scattering (SALS) and SAXS, crystallization behavior of a selected aliphaticaromatic copolyester was further explored. Selected copolyester crystallizes in the form of thin fibrous crystals, few nanometers thick, which is the main factor influencing the depression of its melting temperature.
\end{abstract}

Keywords: aliphatic-aromatic copolyesters, composition, properties

PACS: $82.35 . \mathrm{Jk}$

\section{INTRODUCTION}

With the growing awareness of environmental problems induced by plastic waste great interest has been given to biodegradable polymeric materials. Until now, aliphatic polyesters have been found to be the most attractive biodegradable polymers. Those polymers are susceptible to microbial attack due to the specific ester structures in the main chain. Although the aliphatic polyesters are available on the market, their thermal and mechanical properties do not favor their usage in many applications. Thus the improvement of thermal and mechanical properties has become an important task.

Aromatic polyesters such as poly(ethylene terephthalate) or poly(butylene terephthalate) possess better thermal and mechanical properties in comparison to aliphatic polyesters. Therefore the incorporation of aromatic units along aliphatic polyester chains appeared to be a good concept to merge biodegradability characteristic for aliphatic polyesters and good thermal and mechanical properties typical for aromatic polyesters. However, aliphatic-aromatic copolyesters (AAC) are biodegradable under the condition of sufficiently short sequences of aromatic units which was shown by Witt et $\mathrm{al}^{1}$. Moreover, Witt et al. ${ }^{2}$ drew a conclusion that AAC remain biodegradable provided that the concentration of aromatic ester comonomers does not exceed $60 \mathrm{~mol} \%$.

The aim of our studies, was to produce AAC, which would optimally combine ability to biodegradation and good physical performance. Therefore, a series of poly(butylene adipate- $c o$-succinate- $c o$-glutarate- $c o$-terephthalate) (PBASGT) copolyesters differing in composition has been synthesized and characterized by ${ }^{1} \mathrm{H}$ NMR spectroscopy, wide- (WAXS), small-angle X-ray scattering (SAXS) and uniaxial drawing.

From the synthesized copolyesters the most promising composition with respect to biodegradability, thermal and mechanical properties was selected for further investigation. The spectroscopy and X-ray methods were complemented by differential scanning calorimetry (DSC), polarizing light microscopy (PLM) and small-angle light scattering (SALS). 


\section{EXPERIMENTAL SECTION}

\section{Copolyesters Synthesis}

The scheme of reactions leading to copolyesters is presented in Figure 1. Copolymers were prepared by a twostep melt polycondensation method.

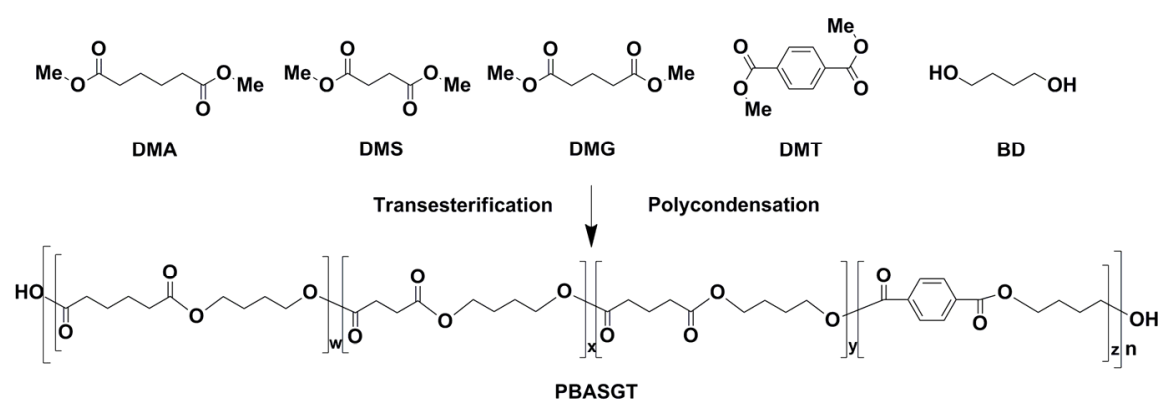

FIGURE 1. Preparation and chemical structure of poly(butylene adipate-co-succinate- $c o$-glutarate-co-terephthalate) copolyester.

A $0.9 \mathrm{dm}^{3}$, stain-less steel reactor equipped with an agitator was used for the synthesis. It was filled with appropriate amount of dimethylterephthalate, esters mixture, butanediol and titanium(IV)butoxide catalyst to obtain a copolyester with required composition. The mixture was heated up to $160^{\circ} \mathrm{C}$ under nitrogen and the ester exchange was proceeded at the temperature rising from $160^{\circ} \mathrm{C}$ to $220^{\circ} \mathrm{C}$ with methanol distillation out of the clave. Then, the second portion of the titanium(IV)butoxide catalyst was added to the reacting mass. The pressure was reduced in step-wise manner to $0.54 \mathrm{hPa}$ and excess of 1,4-butanediol was distilled. The polycondesation reaction proceeded at $250^{\circ} \mathrm{C}$. The obtained copolyesters were extruded out of the reactor after $80 \mathrm{~min}$ in the form of strings which were pelletized.

\section{Experimental Methods}

Copolyesters differing in composition have been synthesized and characterized by ${ }^{1} \mathrm{H}$ NMR spectroscopy, wide(WAXS) and small X-ray scattering (SAXS). Thermal and mechanical properties of the copolyesters were studied by differential calorimetry (DSC), dynamic mechanical thermal analysis (DMTA) and during uniaxial drawing. To have an insight into the mechanism of plastic deformation, deformed specimens of a selected copolyester was studied by SAXS. Polarized light microscopy (PLM), small-angle light scattering and SAXS were used in situ for further crystallization observation in the case of selected copolyester.

\section{RESULTS AND DISCUSSION}

\section{Sequence Distribution Analysis}

So far, it has been recognized that properties of aliphatic-aromatic copolyesters, such as biodegradability and crystallization behavior, are inherently influenced by their macromolecular chain architecture. Thus, to interpret the properties of aliphatic-aromatic copolyesters and occurring phenomena it was necessary to determine the composition and sequence distribution of comonomers. NMR spectra of methylene proton peaks for adipate, succinate, glutarate and terephthalate comonomers depicts the signals related to butylene units which, as Park and Kang ${ }^{3}$ have shown, are not observed in spectra of corresponding homopolymers. Generally, there are sixteen kinds of butylene units (X) distributed in copolyester macromolecules, in which we can distinguish ten homolinkages (AXA, AXS, AXG, SXA, SXS, SXG, GXA, GXS, GXG, TXT) and six heterolinkages (AXT, SXT, GXT, TXA, TXS, TXG). We should also underline that nine of the homolinkages are formed by aliphatic unit (A, S, G) adjacent to butylene unit and one formed by two aromatic units (T). Thus, the observed multiples arise from splitting up butylene proton signals that stand for four potential environments of butylene units, which integrals are changing with respective amount of aliphatic/aromatic units in chains of copolyesters.

Since the BT is asymmetric, the heterolinkages with $\mathrm{T}$ are not commutative, i.e. SXT is not the same as TXS. It concerns also other heterolinkages: AXT and GXT. Hence, the chemical shift for methylene proton of butylene units 
of SXT and TXS are different which is evident from Table 1. Following Park and Kang ${ }^{3}$, we considered four types of sequences. There are two groups of ${ }^{1} \mathrm{H}$ NMR signals, the first in the narrow range of 4.5-4.0 ppm and the second in the narrow range of 2.0-1.5 ppm. Thus, a precise sequence analysis requires a high signal resolution. One can distinguish two ways of analysis: by choosing both groups of signals or by taking into consideration only signals in the range of 4.5-4.0 ppm. We have used the butylene protons related peaks, in the range of 4.5-4.0 ppm, because they are better resolved. In this way the precision of sequence determination should be higher.

TABLE 1. Possible diester environment and chemical shift for PBASGT copolyesters.

\begin{tabular}{lccc}
\hline Type of Dyads & \multicolumn{4}{c}{ AlAl } \\
\hline Symbol & AXA AXS AXG SXA SXS SXG GXA GXS GXG & \\
\hline Chemical Shift $/ \mathrm{ppm}$ & & 4.07 and 1.67 & ArAr \\
\hline Type of Dyads & AlAr & ArAl & TXT \\
\hline Symbol & AXT SXT GXT & TXA TXS TXG & 4.41 and 1.95
\end{tabular}

The integrated intensities of chosen sequences were taken into account in order to determine the copolymer composition and comonomer sequences. There is a way of describing the sequences distribution proposed by Yamadera and Murano ${ }^{4}$, which defines the probability $(P)$ of finding a unit next to an aromatic unit, the average block length $(\bar{L})$ and the degree of randomness $(R)$, by the following equations:

$$
\begin{gathered}
P_{A r}=\frac{\left(f_{A l A r}+f_{A r A l}\right)}{2}+f_{A l A l} \\
P_{A r}=\frac{\left(f_{A l A r}+f_{A r A l}\right)}{2}+f_{A r A r} \\
L_{n A l}=\frac{2 P_{A l}}{\left(f_{A l A r}+f_{A r A l}\right)} \\
L_{n A r}=\frac{2 P_{A r}}{\left(f_{A l A r}+f_{A r A l}\right)} \\
R=\frac{1}{L_{n A l}}+\frac{1}{L_{n A r}}
\end{gathered}
$$

For a mixture of homopolymers $R=0$, the values between 0 and 1 are obtained for a sequential structure, $R$ is 1 for fully random copolymer, and it becomes 2 for strictly alternating distribution.

As expected the peak corresponding to aromatic ring increased smoothly with increasing aromatic units content. In the case of copolyesters with aromatic unit content below $70 \mathrm{~mol} \%$ the structure is random, and above $70 \mathrm{~mol} \%$ becomes closer to alternating.

\section{Thermal Properties}

On the DSC heating traces for copolyesters with aromatic content less than $60 \%$ a broad peak of melting is observed, which with the increase of aromatic units becomes narrower and its peak temperature shifts to higher values. The same phenomena is observed for the crystallization peak. The changes of glass transition temperature together with the increase of aromatic units content was also observed.

\section{Crystal Structure}

Although most of the synthesized copolyesters were found to be nearly random, sharp peaks were observed in WAXS patterns, confirming that only a few units next to each other in macromolecules are sufficient for forming crystals. Furthermore, it was found that the WAXS patterns obtained for copolyesters are similar to that of PBT. Following Alter and Bonart ${ }^{5}$ and Li et al. ${ }^{6}$, we suggest that the crystals of the PBASGT copolyesters are built of triclinic unit cell of PBT. However, it is worth to underline that there are small differences between the diffraction angle positions of respective diffraction peaks of synthesized copolyesters and aromatic homopolymer. 


\section{Stress-Strain Behavior}

Copolyesters with aromatic unit content below 80\% deformed in an uniform manner characteristic for rubbers. With increasing aromatic units content the yield stress point becomes well-defined and the deformation manner changes. For copolyester with $80 \%$ of aromatic content a rubber deformation manner becomes stress-oscillating (or self-oscillating) due to jumping from uniform stable necking to neck propagation form of deformation and reverse. With further increase of aromatic content to $90 \%$ the deformation proceeds in a manner characteristic for semicrystalline thermoplastics with highly localized yielding, and formation of a well-defined neck or by simple brittle breaking, which is characteristic for aromatic homopolymer.

\section{CONCLUSIONS}

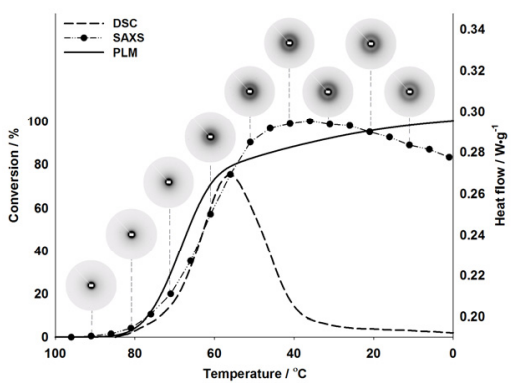

FIGURE 2. SAXS total integral intensity, DSC thermogram and depolarized light integrated intensity recording during PBASGT crystallization at a cooling rate $5^{\circ} \mathrm{C} \mathrm{min}^{-1}$. SAXS scattering patterns illustrate the formation of supermolecular structure during cooling.

Most of the synthesized copolyesters was nearly random copolymer. Surprisingly, it appeared that they are able to crystallize. The increase of aromatic units contents caused an increase in crystallization temperature but did not change the crystallographic structure, in contrary to the results obtained by Li et al ${ }^{5}$. It turned out also that in all the crystallizable copolyesters only butylene terephthalate (BT) blocks are able to crystallize in the crystallographic form characteristic of homopolyper poly(butylene terephthalate). It is worth to underline that there are slight differences in several diffraction peak positions (not all) of synthesized copolyesters and aromatic homopolymer.

From the synthesized copolyesters the most promising composition with respect to biodegradability, thermal and mechanical properties was selected for further investigation. It revealed that PBASGT crystals form spherulitic aggregates filling the entire volume of the material with spherulites of average diameter around $7 \mu \mathrm{m}$, which are formed from few nanometers thick fibrous crystals while the crystallinity reached $20 \%$ (Figure 2).

\section{ACKNOWLEDGMENTS}

This research project has been supported by the European Union Regional Development Fund, Contract No. POIG.0101.02-10-025/09.

Research grant N N209 230638 from Polish Ministry of Science and Higher Education is also acknowledged for partial financial support.

Research project I-20110505 EC Small angle X-ray scattering studies of plastic deformation in semicrystalline homopolymers and DESY Hamburg is also acknowledged for giving the possibility to make in situ measurements.

Research Grant for Young Researchers Development from CMMS PAS 2014 is also acknowledged for partial financial support.

\section{REFERENCES}

1. U. Witt, R. J. Müller, W. D. Deckwer, J Environ Polym Degrad 4, 9-20 (1996).

2. U. Witt, M. Yamamoto, U. Seelinger, R. J. Müller, V. Warzelhan, Angew Chem Int Ed 38, 1438-1442 (1999)

3. S. S. Park, H. J. Kang, Polym J 31, 238-245 (1999)

4. R. Yamadera, N. Murano, Polym Sci 5, 2259-2268 (1967)

5. U. Alter, R. Bonart, Coll \& Polym Sci 254, 348-357 (1976)

6. F. Li, X. Xu, Q. Hao, Q. Li, J. Yu, A. Cao, J Polym Sci 44, 1635-1644 (2006) 